OAI-PMH: http://www.indteca.com/ojs/index.php/Revista Scientific/oai

Ensayo Original / Original Essay

\title{
Gestión de la Gerencia Tecnológica en la Administración Pública
}

Autora: Arisyudith Yelitza Ruiz Ruiz Universidad Fermín Toro, UFT arisyudith@gmail.com

Portuguesa, Venezuela

\section{Resumen}

El propósito del ensayo es reflexionar sobre gestión tecnológica en la administración pública. El proceso metódico utilizado para el acopio de la información que fundamenta este ensayo, se apoya en la revisión documental de algunos esbozos teóricos relacionados con el episteme de la ciencia de la administración y la gerencia para el cumplimiento de los procesos de planeación, organización, dirección, seguimiento y control de las acciones destinadas a alcanzar los objetivos dirigidos a la adquisición, así como la implementación de las tecnologías para el acceso a la información, comunicación, dirigidas al esbozo, desarrollo, elaboración y comercialización de bienes, o la prestación de servicios, asociados los objetivos estratégicos en la atención de los ciudadanos. Se concluyó que la gerencia tecnológica de la administración pública debe responder a las demandas de un mundo globalizado, como componente de la administración estratégica que deben extenderse a los dinamismos de los sistemas informativos y comunicacionales, para dar un salto cualitativo en los modos de relacionarse en los entornos digitales del ciberespacio, lo cual permite la incursión exitosa en la sociedad del conocimiento.

Palabras clave: gestión; tecnología de la información; administración pública; tic. 


\title{
Management of Technological Management in the Public Administration
}

\begin{abstract}
The purpose of the essay is to reflect on technological management in public administration. The methodical process used to collect the information that is the basis of this essay, is based on the documentary review of some theoretical sketches related to the episteme of the science of administration and management for the fulfillment of the processes of planning, organization, management, monitoring and control of actions aimed at achieving the objectives aimed at the acquisition, as well as the implementation of technologies for access to information, communication, aimed at the outline, development, development and marketing of goods, or the provision of services, associated strategic objectives in the attention of citizens. It was concluded that the technological management of public administration must respond to the demands of a globalized world, as a component of strategic management that should be extended to the dynamism of information and communication systems, to make a qualitative leap in the ways of relating in the digital environments of cyberspace, which allows the successful entry into the knowledge society.
\end{abstract} ict.

Keywords: management; information technology; public administration; 


\section{Introducción}

La implementación de las tecnologías en los servicios de la administración pública, ha generado transformaciones, debido a la digitalización de la información que facilita los procesos de comunicación, así como el acceso de los ciudadanos a las transacciones de cualquier índole. Todas ellas deben estar orientadas a desarrollar sus funciones en términos de satisfacer las demandas de cada organización social, mediante la cual se define e implanta las herramientas dirigidas a lograr una gestión de la gerencia capaz de contribuir a la mejora continua de los procesos que se despliegan a través de ella.

La asimilación tecnológica en los procesos productivos de bienes y servicios, está relacionada con una gestión orientada hacia un enfoque de los sistemas administrativos dirigidos a la adopción de los procedimientos innovadores. Estas implementaciones, deben ser transferidas directamente al contexto de cualquier organización en pro de modelar, validar, verificar, mantener la innovación y el conocimiento para desarrollar dichas aplicaciones en la cultura organizacional de instituciones de la administración pública.

A tal efecto, la gestión tecnológica agrega valor a la gerencia por la infinidad de formas organizadas de acceso a la información, comunicación y conocimiento, dirigidos a la construcción colectiva de creencias, perspectivas, conceptos, juicios, expectativas, que permiten la aplicación de nuevas maneras de asimilación de procesos, aunque tenga un objetivo distinto. Por lo tanto, una gestión eficaz debe permitir optimizar las condiciones organizacionales para lograr un equilibrio funcional en la gerencia de la administración pública.

De acuerdo con lo expuesto, el propósito de este ensayo es reflexionar acerca de la gestión que involucra la gerencia tecno-administrativa pública. El proceso metódico utilizado para el acopio de la información que fundamenta este ensayo, se apoya en la revisión documental de algunos esquemas 
teóricos relacionados con los constructos de estudio, los cuales se presenta a continuación.

\section{Argumentos del Análisis Reflexivo}

Las tendencias sociales, políticas administrativas durante las últimas décadas, han creado nuevas dinámicas en las organizaciones, como alternativas gerenciales para adaptarse a la dinámica compleja del crecimiento de la sociedad. De ahí que, la gestión tecnológica se concibe en la actualidad, desde varios puntos enfoques que la ubican en las políticas públicas referidas a innovación como una parte importante de los planes estratégicos en el desarrollo integral de cada nación, para ir adecuándose a los cambios científicos y técnicos que se despliegan en las instituciones públicas.

Cada vez más, el acceso a los recursos tecnológicos facilita una mejoría en las funciones desplegadas en las instituciones, sobre todo en las que se incorpora una estrategia tecnológica dirigida a la identificación, evaluación, selección para la adquisición eficaz de recursos que serán utilizados eficientemente por los gerentes en los procesos que despliegan en la administración pública.

Como expone Restrepo (2000): al gerente, le incumbe los procesos destinados a dirigir "una mirada al entorno, de modo que la organización pueda generar desarrollo: tomar recursos y producir más recursos. Divide las competencias de los términos gestión, gerencia y administración" (pág. 15). Lo cual sugiere que la tarea, debe ser concebida como una parte de las acciones la administración que permiten alcanzar las funciones vitales de la organización.

De acuerdo con los planteamientos de Drucker (2012): la gerencia cumple múltiples funciones, entre ellas las de "coordinar todos los recursos a través de los procesos de planeamiento, organización, dirección y control, con el fin de lograr los objetivos establecidos" (pág. 97). Asimismo, el manejo de la 
gestión tecnológica representa parte de la innovación en las acciones que exploran las propuestas que involucran la creatividad e innovación en las gestiones ajustadas a las necesidades del ambiente laboral para que sea lo más efectiva posible.

Si se considera que las innovaciones tecnológicas, han cambiado las respuestas organizacionales a los requerimientos de la sociedad; la gestión gerencial es determinante para lo que Barroso (2000): sugiere como un proceso organizativo de toma de decisiones que, además debe lidiar con la incertidumbre y complejidad de los sistemas tecno administrativos para añadir valor a planes estratégicas de los gerentes. (pág. 18).

Los planes estratégicos de los gerentes en las organizaciones, deben estar orientados a garantizar el suministro de los recursos, entre ellos los humanos, materiales y equipos, así como los económicos, destinados al funcionamiento técnico-administrativo, destinados a satisfacer los requerimientos que engloben todos los aspectos de la vida laboral de los ciudadanos, para atender a las exigencias de información, comunicación y conocimiento destinados a la gestión tecnológica en la administración pública.

La gestión tecnológica, representa para Jaimes, Ramírez, Vargas y Carrillo (2011a): un conjunto de procesos "claves para el cumplimiento de los objetivos estratégicos de una organización; con el objetivo de generar productos y/o servicios competitivos a partir del aprovechamiento de su cantidad tecnológica" (pág. 4). Éstos deben estar vinculados en forma y contenido social, técnico, administrativo y económico, entre otros, en términos de sustentabilidad de las organizaciones públicas, basados en los requerimientos de los ciudadanos, como respuesta de la responsabilidad en la sociedad del conocimiento.

Expone Castells (2002): que la sociedad del conocimiento, "se trata de las condiciones de generación de conocimiento y procesamiento de información han sido sustancialmente alteradas" (pág. 184). Desde esta 
perspectiva estratégica, la gestión tecnológica en la administración pública, no escapa a esta realidad; razón por la cual, se orienta en la obtención de los activos técnicos a través de un conjunto de acciones que conllevan a la innovación de los tramites que se realizan en estas instituciones, para la satisfacción de los requerimientos de los ciudadanos.

Igualmente, los esbozos de Imbernón (2013): sobre la sociedad del conocimiento, se acoge a la idea tecnológica, como una manera de identificar cada uno de los componentes, donde se articulan los saberes generados de las demandas de información, comunicación, conocimientos, concebidos por los avances sociales y productivos, que necesitan de todos los entes de la administración pública para poder establecer un mejor afianzamiento desde la integración participativa de los ciudadanos. (pág. 205).

La sociedad del conocimiento como se le denomina actualmente, despliega en la gestión tecnológica el acrecentamiento de la capacidad informacional, comunicativa, así como generadora de conocimientos, que se amplían con la apropiación de las redes sociales representadas por WhatsApp, Instagram, Facebook, entre otras aplicaciones de la Web, que favorecer la cooperación ciudadana, sobre todo en la administración pública.

Además, la Fundación COTEC para la Innovación Tecnológica (COTEC, 2003a), señala que las gestiones incluyen:

Todas aquellas actividades que capacitan a una organización para hacer el mejor uso posible de la ciencia y la generada, tanto de la forma externa, como interna. Este conocimiento conduce hacia una mejora de sus capacidades de innovación, que ayuda a promocionar la eficiencia y la eficacia de la organización para obtener ventajas competitivas (pág. 46).

En este orden de ideas, en los entes gubernamentales públicos la gerencia debe apoyarse en las normativas legales vigente, sobre todo en aquellas gestiones tecnológicas destinadas a la administración eficiente de los servicios a los ciudadanos, orientando las acciones tecnológicas estratégicas 
para lograr el desempeño de las normas y procedimientos que rigen las actuaciones laborales en estas instituciones.

Esta orientación organizativa, resulta relevante en el establecimiento de una gestión tecnológica estratégica, que haga viable las decisiones tácticas y operativas que garanticen los cambios a nivel local con miras a la incorporación del conocimiento organizacional, lo cual contribuiría al despliegue de la capacidad informativa y comunicacional en la visión de una sociedad del conocimiento, que agregaría valor a gestión tecnológica en la administración pública.

Al respecto, Maya (2006): explica que el valor de la gestión tecnológica las políticas de funcionamiento, debe están ligadas a los procedimientos esenciales para la producción de acciones destinadas a la generación de bienes o servicios informativos, comunicativos y del conocimiento, sobre la base de un conjunto de acciones operativas de los gerentes, enfocadas al desarrollo eficiente de los procesos técnicos-administrativos (pág. 116).

Al respecto, Certo S-Piter (2001): sostiene que la tecnología, "puede ser utilizada en forma sistemática para el diseño, desarrollo, fabricación y comercialización de productos o para la prestación de servicios, incluyendo la aplicación de las técnicas asociadas a la gestión" (pág. 45). Como herramienta administrativa, ha traído consigo un proceso de reflexión sobre la importancia de las competencias digitales de los gerentes en su desempeño para la conducción organizacional en la administración pública.

Como derivada, los gerentes en las organizaciones deben desplegar una capacidad de gestión tecnológica, que Jaimes, Ramírez, Vargas y Carrillo (2011b), señalan: se componen de cinco (5) procesos: (a) diseño de la estrategia, (b) planificación del desarrollo, (c) capacitación, (d) adquisición de recursos, y (e) transferencia de tecnología. (pág. 104).

La transferencia tecnológica, según la Fundación COTEC para la Innovación Tecnológica (COTEC, 2003b): señala que representa el "capital 
intelectual y del know-how (saber-hacer) entre las organizaciones, con la finalidad de su utilización en la creación y utilización de productos y servicios" (pág. 10). Implica un intercambio de habilidades, conocimientos técnicos, entre otros destinada al aprovechamiento de los avances tecno científicos, de tal modo de convertirlos en servicios eficientes para las instituciones públicas que los implementan.

Además, la transferencia tecnológica en el ámbito de las actividades de la administración pública, representa un proceso de incorporación de gestiones dirigidas al manejo de información de los sistemas de selección, procesamiento y difusión comunicativa para hacer asimilable el uso de los conocimientos gerenciales en las organizaciones.

De esta manera, los gerentes en la gestión o administración pública, deben tener claros los aspectos relacionados con la incorporación de las Tecnologías de Información y Comunicación (TIC), las cuales señalan Yánez y Villatoro (2005): "representan los instrumentos de apoyo a la gestión de la política social (...) como elementos de trabajo en el desarrollo de la organización local" (pág. 12). Por ello el elemento más representativo, es sin duda, el ordenador y más específicamente, la Internet, lo que ha permitido dar un salto cualitativo de gran magnitud, que ha cambiado los modos de conocer y relacionarse que permite la incursión exitosa en la sociedad del conocimiento.

La incorporación de las (TIC) en la gestión tecnológica en la administración pública, permite entre otros aspectos, la búsqueda de información de modo rápido, oportuno, organizado de forma más eficiente y efectiva con los requerimientos comunicativos que ayudan a la simplificación de los procesos de transferencia tecnológica de los procedimientos técnicos y administrativos de la organización pública.

Cabe señalar los planteamientos de Münch y García (2004): sobre los procedimientos tecno-administrativos como instrumentos utilizados como 
apoyo a la realización del quehacer cotidiano organizacional, debido a posibilidad de determinar "el orden lógico que debe seguir las actividades" (pág. 5). Estas acciones en la organización se desarrollan en forma metódica y son fundamentales en las operaciones de acuerdo con las funciones desempeñadas en los ámbitos laborales.

Por lo tanto, el gerente en la administración pública en muchos casos cumple diversas funciones que le exige desenvolverse como administrador, supervisor, contralor, auditor, entre otras actuaciones inherentes a su cargo. En este contexto gerencial, en estas organizaciones, también se desarrollan acciones relacionadas con la dirección y control, las cuales se ven impregnadas por la burocracia de los procesos tecno-administrativos que se desarrollan en ellas.

Los procesos tecno-administrativos que se despliegan en la gestión tecnológica persiguen integrar los objetivos estratégicos de los entes públicos, en términos de posesionarse en una actitud proactiva que permita introducir cambios favorables a los procesos informativos y comunicativos destinados a incrementar la productividad, así como el desempeño laboral eficiente en las organizaciones de la administración pública.

Siguiendo estas ideas, la Constitución de la República Bolivariana de Venezuela (1999), en el Artículo 141, establece que la administración pública está al servicio de los ciudadanos, para lo cual se fundamenta en los principios de transparencia, rendición de cuentas y responsabilidad en el ejercicio de la función pública; Asimismo, en el Artículo 142, señala que las instituciones u organizaciones públicas responden a los intereses de la sociedad; dichas normativas se explican en la Ley Orgánica de la Administración Financiera del Sector Público (LOAFSP, 2005): expone los procedimientos para el funcionamiento de las instituciones oficiales, Contemplado en el artículo 1.

Sin embargo, los procesos fundamentados en las normativas jurídicas de cada organización pública, algunas veces no tífica claramente las funciones 
fundamentales que debe realizar en el gerente, en pro de lograr el alcance de los objetivos establecidos, de manera de lograr la prestación de servicio con eficaz y eficiencia, para ello, se requiere de cierta flexibilidad en el desempeño de actividades en la sociedad del conocimiento y del bienestar de la colectividad.

Por lo tanto, dentro de esta dinámica el gerente en la administración pública en muchos casos cumple diversas funciones que le exige desenvolverse como administrador, supervisor, contralor, auditor, entre otras actuaciones inherentes a su cargo. En este contexto gerencial, en estas organizaciones, también se desarrollan acciones relacionadas con la dirección y control, las cuales se ven impregnadas por la burocracia de los procesos tecno-administrativos que se desarrollan en ellas.

Los planes estratégicos de desarrollo sustentable, deben estar orientados a garantizar el suministro de los recursos requeridos para satisfacer los requerimientos de los ciudadanos. Éstos deben estar vinculados en forma y contenido, a los aspectos sociales, por supuesto debe contemplar lo económico en términos de sustentabilidad a través de una planificación basada en los requerimientos de los ciudadanos, como respuesta de la responsabilidad en la sociedad del conocimiento.

De esta manera, las instituciones públicas tienen a su cargo un cuerpo administrativo, conformado por un personal que desempeña actividades dentro de la organización desde las cuales, tienen una responsabilidad social en la sociedad del conocimiento en pro del bienestar de la colectividad.

\section{Consideraciones Finales}

En respuesta al propósito de este ensayo, la gestión tecnológica debe estar destinada a elevar la competitividad y el aprovechamiento de las capacidades de respuesta de la administración pública, tanto en el acceso de la información, como en las acciones comunicativas indispensables para 
generar conocimientos pertinentes con una sociedad del conocimiento cada día más globalizada.

Por ello, en la sociedad del conocimiento, la administración pública, representa el acceso de la información y las vías de comunicación de los ciudadanos a través de los avances tecnológicos, donde se articulen los saberes en concordancia con las demandas sociales de los sectores productivos y comunitarios.

La revisión de los planteamientos teóricos que fundamentan las reflexiones sobre la gestión tecnológica como estrategia de los gerentes de la administración pública, como mecanismos estratégicos para planificar la conducción de las organizaciones, como un todo integrado para la prestación de bienes y servicios.

La utilización de las Tecnologías de Información y Comunicación (TIC); requiere una gerencia capaz de desarrollar en el talento humano que labora en las organizaciones de la administración pública, las competencias digitales destinadas al uso de las redes sociales para favorecer la transferencia tecnológica e informativa en estas instituciones.

Las gestiones tecnológicas, deben estar vinculados en forma y contenido, a los aspectos sociales, por supuesto debe contemplar lo económico en términos de sustentabilidad a través de una planificación basada en los requerimientos de los ciudadanos, como respuesta a las exigencias que se originan en la sociedad del conocimiento.

De este modo, la gestión tecnológica en la administración pública, representa las áreas relacionadas con la gerencia de estas instituciones requiere de personal altamente especializado, capaz de mediar las acciones a través de las (TIC) para que las acciones individuales, así como colectivas en la cotidianidad, se desarrollen de manera eficaz y eficiente.

Finalmente, se concluyó que la gestión de la gerencia tecnológica en la administración públicas debe responder a las demandas de un mundo 
globalizado, como componente organizacional estratégica deben extenderse a los sistemas de información, comunicación y acceso al conocimiento, para dar un salto cualitativo en los modos de relacionarse en los entornos digitales del ciberespacio, lo cual permite la incursión exitosa en la sociedad del conocimiento.

\section{Referencias}

Barroso, M. (2000). Meditaciones Gerenciales. Venezuela: Galoe.

Castells, M. (2002). La Dimensión Cultural de Internet. Barcelona, España: Universitat Oberta de Catalunya, UOC. Recuperado de:

http://www.uoc.edu/culturaxxi/esp/articles/castells0502/castells0502.ht $\underline{\mathrm{ml}}$

Certo S-Piter, J. (2001). Dirección Estratégica. Madrid: Ediciones Irwin. Constitución de la República Bolivariana de Venezuela, CRBV (1999). Gaceta Oficial $N^{\circ}$ 36.860. Jueves, 30 de diciembre. Caracas, Venezuela: Asamblea Nacional Constituyente. Recuperado de:

http://www.inpsasel.gob.ve/moo doc/ConstitucionRBV1999-ES.pdf

Drucker, P. (2012). Principios de la Organización Administrativa. 2da. edición. México: Prentice Hall.

COTEC (2003a,b). Transferencia Tecnológica. Madrid, España: Fundación COTEC para la Innovación Tecnológica.

Imbernón, F. (2013). La Profesión Docente en la Globalización y la Sociedad del Conocimiento. Barcelona, España: Editorial Aguilar. LOAFSP (2005). Ley Orgánica de la Administración Financiera del Sector Público. Gaceta Oficial № 38.198. Martes, 31 de mayo. Caracas, Venezuela Caracas, Asamblea Nacional de la República Bolivariana de Venezuela.

Maya, R (2006). Gestión Tecnológica en las Universidades Públicas en el Departamento del Cesar. Colombia: Editorial UNICESAR. 
Münch, L., \& García, J. (2004). Fundamentos de Administración. México: Trillas.

Jaimes, M., Ramírez, D., Vargas, A., \& Carrillo, G. (2011a,b). Gestión Tecnológica: Conceptos y Casos de Aplicación. Bucaramanga, Colombia: Universidad Industrial de Santander.

Restrepo, G. (2000). El concepto y Alcance de la Gestión Tecnológica. Revista Facultad de Ingeniería, (21), págs. 11, ISSN: 2422-2844. Medellín, Colombia: Universidad de Antioquia.

Yánez, M., \& Villatoro, P. (2005). Las Nuevas Tecnologías de la Información y de la Comunicación (TIC) y la institucionalidad social. Hacia una gestión basada en el conocimiento. Santiago de Chile: División de Desarrollo Social. Naciones Unidas CEPAL. 
Nacida en Guanare Estado Portuguesa, Venezuela.

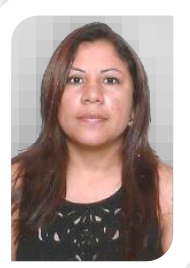
Licenciada en Administración mención: Recursos Materiales y Financieros (EBAP). Especialista en Gerencia Integral en Control Fiscal y Auditoria. Especialista en Docencia Universitaria con Maestría en Gerencia Empresarial. 